\title{
Dephosphorization of Manganese Ore Raw Materials
}

\section{Veniamin Ya. Dashevskii ${ }^{1}$, Alexander L. Petelinn ${ }^{2}$, Aleksandr A. Aleksandrov ${ }^{1}$, Larisa A. Polulyakh², and Dmitriy B. Makeev²}

${ }^{1}$ Institute of Metallurgy and Materials Science. A. A. Baykova RAS, Moscow, Russian

${ }^{2}$ National University of Science and Technology "MISiS", Moscow, Russian

\section{Abstract}

The dephosphorization of manganese ores and concentrates in a reducing atmosphere is thermodynamically analyzed. It is shown that phosphorus can completely pass to a gas phase in a closed reaction system in a wide temperature range (1273-2073 K) at the amounts of a reducing gas (CO) that exceed the stoichiometric minimum required for reduction reactions. The gaseous products of reduction is found to contain phosphorus

Corresponding Author: Veniamin Ya. Dashevskii vdashev@imet.ac.ru

Received: 5 February 2019 Accepted: 6 March 2019 Published: 17 March 2019 Publishing services provided by Knowledge E

(c) Veniamin Ya. Dashevskii et al. This article is distributed under the terms of the Commons Attribution License, which permits unrestricted use and redistribution provided that the original author and source are credited.

Selection and Peer-review under the responsibility of the NIOKR-2018 Conference Committee.

\section{G OPEN ACCESS}

in the form of mainly polyatomic "heavy" molecular oxides, which can decrease the real effect of dephosphorization as compared to that obtained by equilibrium calculations because of kinetic factors. A thermodynamic simulation of a flow reaction system shows that almost complete transition of phosphorus to light gaseous substances $\left(P O, P_{2}\right)$ is thermodynamically possible at the temperatures that are close to the technological operation temperatures. This transition is provided by the ratio of the rate of formation of volatile phosphorus-containing substances to the rate of their removal from reaction regions.

Keywords: manganese ores, manganese concentrates, phosphorus, carbon monoxide, reduction

\section{Introduction}

The manganese ore reserves in the Russian Federation are estimated as $\mathbf{2 8 3 . 2}$ million $t$ [1]; however, the production of manganese ores is very weak because of their low quality. About $80 \%$ of the reserves are represented by carbonate ores with an average manganese content of $20 \%$ and a high phosphorus content $(0.2-0.3 \%$ or more), the rest - oxide and mixed ores containing $23-26 \% \mathrm{Mn}$. Therefore, these ores should be subjected to dephosphorization for standard manganese ferroalloys with the required phosphorus content to be made from the manganese ores and concentrates of the deposits located in the Russian Federation. 
A pyrometallurgical dephosphorization method, in which gaseous carbon monoxide is a reducer, was developed [2]. Phosphorus is reduced from an oxide melt by gaseous carbon monoxide $(\mathrm{CO})$ blown through an oxide manganese-containing melt rather than by solid carbon in order to dephosphorize manganese ore melts and to eliminate manganese losses with a by-product metal. It is reasonable to study the dephosphorization of manganese-containing products in a $\mathrm{CO}$-containing reducing atmosphere, since manganese is not reduced by this gaseous reducer in a wide temperature range of 973-2073 $\mathrm{K}$, which is evidenced by the temperature dependence of the standard Gibbs energy for the reaction [3]:

$$
\begin{gathered}
(\mathrm{MnO})+\mathrm{CO}(\mathrm{g})=\mathrm{Mn}(\mathrm{s}, \mathrm{l})+\mathrm{CO}_{2}(\mathrm{~g}), \\
\Delta G_{T}^{\circ}=124,745-1.84 T, \mathrm{~J} / \mathrm{mol} .
\end{gathered}
$$

The equilibrium constant of reaction (1) is $K_{(1)}<10^{-7}$ for any real process temperatures. The following reaction can occur when manganese ore and concentrate melts interact with gaseous carbon monoxide [3]:

$$
\begin{gathered}
1 /{ }_{5}\left(\mathrm{P}_{2} \mathrm{O}_{5}\right)+\mathrm{CO}(\mathrm{g})=1 /{ }_{5} \mathrm{P}_{2}(\mathrm{~g})+\mathrm{CO}_{2}(\mathrm{~g}) \\
\Delta G_{T}^{\circ}=34,268-11.38 \mathrm{~T}, \mathrm{~J} / \mathrm{mol} .
\end{gathered}
$$

We now analyze the thermodynamic of reduction of phosphorus in raw materials (ore, concentrate) by a gaseous reducer. The reduction of phosphorus can be analyzed with allowance for a number of factors, the most important of which are temperature and the relative amount of a reducing gas in the reaction zone. The total pressure in the process is taken to be constant, $0.1 \mathrm{MPa}$ (1 atm).

\section{First Level of Consideration}

The reducing process is assumed to occur in a closed system, which means that the law of conservation of matter holds true for each chemical element in the system. No substances penetrate into the system or leave it during the entire time interval under study. The process is assumed to be based on reaction (2) of $\mathrm{P}_{2} \mathrm{O}_{5}$ reduction by a gaseous reducer. This version corresponds to the "heaviest" reduction process, since the strongest (most stable) oxide among the phosphorus oxides serves as an initial 
phosphorus-containing substance. Moreover, no additional ways of reduction with possible formation of gaseous phosphorus oxides are implied. The presence of other components, which can facilitate the recovery of phosphorus from a charge, in the reaction zone is also not taken into account.

Table 1 gives the values of equilibrium constant $K_{(2)}$ of reaction (2) calculated in the temperature range $1273-2073 \mathrm{~K}$ and the calculated values of dephosphorization $X_{P}$ in mole fractions (percentage) of phosphorus having passed to a gas phase as functions of temperature and the amount of a reducing gas in the initial state. The calculation results demonstrate that the degree of dephosphorization increases with temperature: up to $1573 \mathrm{~K}$, the dephosphorization rate is the dephosphorization rate is $16 \%$ per 100 $\mathrm{K}$. When the temperature increases further, the dephosphorization rate decreases to $7-$ $8 \%$. On average, the rate of phosphorus transfer to a gas phase is close to $12 \%$ (per 100 $\mathrm{K}$ when temperature increases) over the entire temperature range.

An increase in the amount of the reducing gas (CO) weakly affects the process. As the parameter that characterizes the relative amount of $\mathrm{CO}$ in the system, we chose $\lambda=n_{C O} / n_{P}$, i.e., the ratio of the number of $\mathrm{CO}$ moles in the system to the number of phosphorus moles. The degree of dephosphorization at 1573-1773 K increases by at most $5 \%$ only when carbon monoxide is introduced in the system in the amount that exceeds the amount of phosphorus by a factor of $20(\lambda=20)$.

These calculation results demonstrate that the reduction of $\mathrm{P}_{2} \mathrm{O}_{5}$, which is present in the initial concentrate, by gaseous carbon monoxide with allowance for the formation of only one phosphorus-containing substance-product does not lead to a positive result. The degree of dephosphorization performed according to this mechanism is insignificant.

TABLE 1: Results of equilibrium calculation of the $\mathrm{P}_{2} \mathrm{O}_{5}$ reduction by carbon monoxide.

\begin{tabular}{|l|l|l|l|l|l|l|l|l|l|}
\hline Parameter & \multicolumn{8}{|c|}{ Temperature, K } \\
& $\mathbf{1 2 7 3}$ & $\mathbf{1 3 7 3}$ & $\mathbf{1 4 7 3}$ & $\mathbf{1 5 7 3}$ & $\mathbf{1 6 7 3}$ & $\mathbf{1 7 7 3}$ & $\mathbf{1 8 7 3}$ & $\mathbf{1 9 7 3}$ & $\mathbf{2 0 7 3}$ \\
\hline $\mathbf{H}_{T}^{\circ}, \mathrm{J} / \mathrm{mol}$ & 19,781 & 16,643 & 17,278 & 16,367 & 15,229 & 14,091 & 12,953 & 11,815 & 10,677 \\
\hline$K_{(2)}$ & 0.154 & 0.195 & 0.244 & 0.286 & 0.334 & 0.384 & 0.435 & 0.486 & 0.538 \\
\hline$X_{P}(\lambda=1), \%$ & 4.4 & 5.0 & 5.7 & 6.2 & 6.7 & 7.2 & 7.8 & 8.1 & 8.6 \\
\hline$X_{P}(\lambda=20), \%$ & 4.3 & 5.1 & 5.7 & 6.5 & 6.9 & 7.5 & 7.9 & 8.3 & 8.7 \\
\hline
\end{tabular}




\section{Second Level of Consideration}

We again analyze the closed system. Recall that the real interaction of a manganese concentrate with a reducing atmosphere includes a large number of parallel chemical reactions, which result in the appearance of gaseous phosphorus $\mathrm{P}_{2}$ and other gaseous molecules containing phosphorus atoms $\left(\mathrm{P}, \mathrm{P}_{3}, \mathrm{P}_{4}, \mathrm{P}_{6}\right.$, etc.; oxides $\mathrm{PO}, \mathrm{PO}_{2}, \mathrm{P}_{2} \mathrm{O}_{3}, \mathrm{P}_{2} \mathrm{O}_{4}$, $\mathrm{P}_{3} \mathrm{O}_{6}$, etc.). In addition, the real system contains manganese oxides, which are the main components of the concentrates used for the production of ferromanganese; iron oxides; and various compounds of other by-product metals (aluminum, calcium, magnesium).

A computer simulation is required to perform a grounded thermodynamic forecast of the dephosphorization of raw materials for the production of ferromanganese in a complex multicomponent system, where a large number of chemical processes occur simultaneously. In this work, we use the Ivtantermo thermodynamic software package to analyze dephosphorization under equilibrium conditions.

The initial composition included all main components of the concentrate and the gaseous atmosphere of the reaction zone except for the elements and compounds that do not affect the reduction of phosphorus. We first carried out a preliminary series of calculations to optimize the initial composition and then performed a thermodynamic simulation of the dephosphorization as a function of temperature and the relative amount of carbon monoxide in the system $(\lambda)$. In Figure 1, we present the distributions of phosphorus-containing gaseous substances in the system under study versus temperature at various values of parameter $\lambda$.

The following conclusions can be drawn from these distribution curves:

(i) Phosphorus that is present in an oxidized form in the initial concentrate almost fully transforms into a gaseous form as the molecules of gaseous phosphorus oxides, which have various stoichiometric compositions and, correspondingly, various molecular masses, at any given temperature and amount of the reducing gas (CO). In this case, the fraction of pure phosphorus molecules, which contain different numbers of phosphorus atoms, in the gas phase is insignificant, at most $0.1 \%$ (in mole units). The differences in the total degrees of transition for all chosen conditions are very small and may be neglected in a first approximation. Thus, almost 100\% (99.5-99.9\%) of oxidized phosphorus should be gasified from the bound state in the condensed substance of the concentrate under equilibrium conditions. 
(ii) The component composition of the phosphorus-containing gas atmosphere is substantially different for different temperatures and amounts of $\mathrm{CO}$ in the system. The higher the temperature, the larger part of phosphorus is in compounds with a small molecular mass (see Figure 1). The same effect was observed when the CO content in the system increased ( $\lambda$ increased). For example, at a temperature of $1573 \mathrm{~K}$, the fraction of phosphorus in the form of "light" molecules is only $0.84 \%$ (in mole units) at the amount of $\mathrm{CO}$ that is close to the amount of gaseous phosphorus $(\lambda=1.33)$, and the fraction of phosphorus increases to $35 \%$ at the same temperature and a significant increase in the amount of $\mathrm{CO}(\lambda=16.67)$.
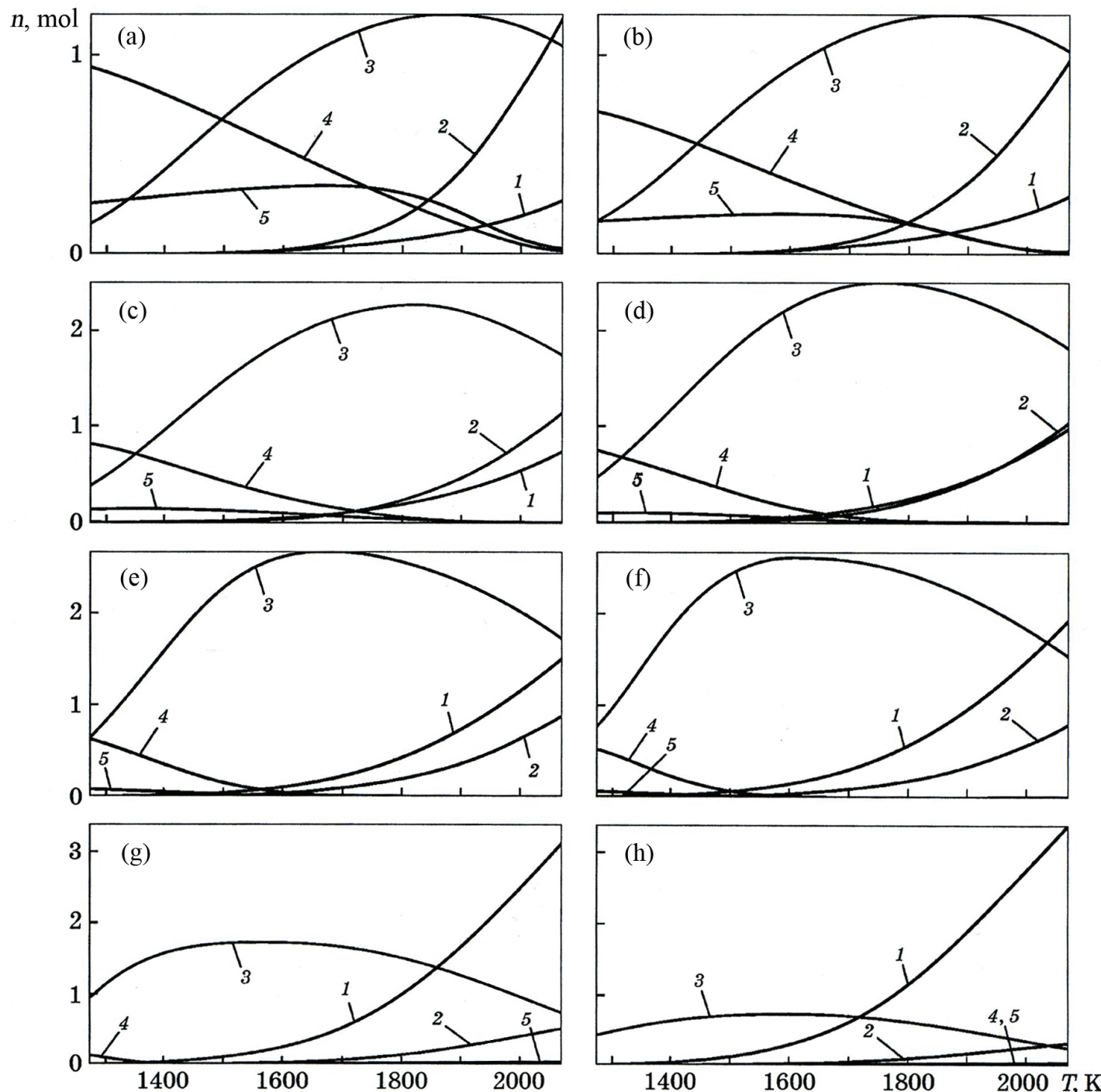

Figure 1: Temperature dependences of the distributions of phosphorus-containing gas components ( $(1) \mathrm{PO}$, (2) $\mathrm{PO}_{2}$, (3) $\mathrm{P}_{2} \mathrm{O}_{3}$, (4) $\mathrm{P}_{4} \mathrm{O}_{9}$, (5) $\mathrm{P}_{4} \mathrm{O}_{10}$ ) at a relative amount of carbon monoxide $\lambda=$ (a) 0.67 , (b) 0.83 , (c) 1.33 , (d) 1.67 , (e) 2.50 , (f) 3.33 , (g) 8.33 , and (h) 16.67 in the system. 
To interpret the calculation results, it is convenient to conventionally divide the entire spectrum of phosphorus-containing gaseous substances into the following three groups: group $A$, substances with a small molecular mass (light volatile $P O, P_{2}$ ); group $B$, substances with a medium molecular mass (medium volatile $\mathrm{P}_{2} \mathrm{O}_{3}, \mathrm{P}_{2} \mathrm{O}_{4}, \mathrm{P}_{3} \mathrm{O}_{6}, \mathrm{P}_{4} \mathrm{O}_{7}, \ldots$ ); and group $C$, substances with a large molecular mass (heavy volatile $\mathrm{P}_{4} \mathrm{O}_{9}, \mathrm{P}_{4} \mathrm{O}_{10}$ ). Table 2 gives the fractions of phosphorus (mol \%) in the substances of groups $A, B$, and $C$ when parameter $\lambda$ changes from 0.67 to 16.67 at $T=1573 \mathrm{~K}$.

TABLE 2: Phosphorus distribution between the gaseous substances of groups $A-C$ in the products of carbon monoxide reduction at $T=1573 \mathrm{~K}$.

$\begin{aligned} & \lambda \text { - relative fraction of } \mathrm{CO} \text {, } \\ & \text { (mole fraction) }\end{aligned}$
0.67
1.33
1.67
2.5
3.33
8.33
16.67

\begin{tabular}{|c|c|}
$\begin{array}{c}\text { A - (light } \\
\text { volatile), \% }\end{array}$ \\
0.5 \\
\hline 0.8 \\
1.2 \\
\hline 2.8 \\
\hline 5.2 \\
22.0 \\
\hline 35.1
\end{tabular}

\begin{tabular}{|c|}
\hline $\begin{array}{c}\text { B - (medium } \\
\text { volatile), \% }\end{array}$ \\
\hline 84.5 \\
\hline 92.0 \\
\hline 94.3 \\
\hline 96.0 \\
94.4 \\
77.9 \\
\hline 64.9
\end{tabular}

C - (heavy
volatile), \%
15.0
7.0
4.5
1.2
0.4
0.1
$10^{-4}$

We assume that the results (and compositions) predicted by equilibrium thermodynamic calculations are only incompletely reached in the real system. Substances (molecules) with a small molecular mass and a small number of atoms per molecule can more easily (and faster) form and be more reliably conserved under variable external conditions. The rate of formation of "heavy" molecules (group B and, especially, C) is usually lower than that of "light" molecules (group A) and their stability is also lower, since the probability of breaking interatomic bonds and molecular decomposition is high when a molecule has a large number of atoms. Therefore, a thermodynamic prediction of the complete phosphorus transition to a gas phase can only be partly actualized because of kinetic reasons.

This conclusion is supported by the experimental results on the phosphorus reduction by carbon monoxide for manganese-containing concentrates [4]. The degree of dephosphorization reached in that work does not exceed $80-90 \%$ and it is even lower in some cases. A number of additional factors have to be taken into account to obtain the results that better correspond to the system operating under real working conditions. 


\section{Third Level of Consideration}

The openness of the system should be taken into account to approach its real behavior closer. The molecules of the gas substances-the products of phosphorus reductionhave a high mobility and, hence, leave the reaction system; that is, the reaction zone is a flow reactor. Constant motion of the reaction products from the reaction zone should shift reactions toward product formation. This behavior of processes can be simulated if the entire process is divided into conventional time intervals T. An equilibrium calculation terminates after time $\mathrm{T}$ for each interval (calculation step). The next step is calculated with allowance for the changes in the concentrations of the initial substances that took place at the previous step, and the amount of products is assumed to be zero. A sequential calculation for $n$ steps would lead to a gradual decrease in the amount of phosphorus in the initial substance until it reaches the value comparable with real experimental values.

Using this scheme, we performed a numerical simulation at a temperature of $1573 \mathrm{~K}$. The initial main component composition for the beginning of the process was identical to that used for the second-level equilibrium calculation. Stable substances with the minimum molecular mass, namely, $\mathrm{PO}$ and $\mathrm{P}_{2}$, were assumed to form during reduction. Parameter $\lambda$ was taken to be minimal (Table $2, \lambda=0.67$ ). The degree of equilibrium phosphorus transition to a gas phase was determined for each of the seven calculation steps (Table 3). The calculation was stopped at a total dephosphorization of $>95 \%$.

TABLE 3: Numerical simulation of the degree of dephosphorization in an open phosphorus-containing system at $T=1573 \mathrm{~K}$.

\begin{tabular}{|l|c|c|}
\hline Step number & $\begin{array}{c}\text { Fraction of } \\
\text { phosphorus in } \\
\text { oxide phase, \% }\end{array}$ & $\begin{array}{c}\text { Degree of dephos- } \\
\text { phorization, \% }\end{array}$ \\
\hline 1 & 80.3 & 20.7 \\
\hline 2 & 61.3 & 38.7 \\
\hline 3 & 43.0 & 57.0 \\
\hline 4 & 25.3 & 74.7 \\
\hline 5 & 12.7 & 87.3 \\
\hline 6 & 6.3 & 93.7 \\
\hline 7 & 0.4 & 99.6
\end{tabular}

The results of numerical simulation demonstrate that almost complete transition of phosphorus to a gas phase, which consists of light and stable phosphorus-containing substances, is possible in the flow system even at a small relative amount of $\mathrm{CO}(\lambda=0.67$, 
which corresponds to $n=4 \mathrm{~mol}$ reducing gas (which is present during the entire reduction of raw materials containing $\left.3 \mathrm{~mol} \mathrm{P}_{2} \mathrm{O}_{5}\right)$ ). Here, the degree of dephosphorization is close to $100 \%$. It should be noted that this result was obtained during an equilibrium step-by-step calculation, which means that equilibrium is reached in the reaction system in time $\mathbf{T}$ at each step.

The validity of these results depends on the reduction kinetics and the rate of removal of gas products from the reaction zone, which can only experimentally be shown.

\section{Conclusions}

1. An equilibrium calculation of the degree of gas dephosphorization showed that phosphorus can completely pass to a gas phase in a closed reaction system in a wide temperature range (1273-2073 K) at the amounts of a reducing gas (CO) that exceed the stoichiometric minimum required for reduction reactions.

2. It was also found that, in the lower part of the temperature range under study, the gaseous products of reduction contain phosphorus in the form of mainly polyatomic heavy molecular oxides, which can decrease the real effect of dephosphorization as compared to that obtained by equilibrium calculations because of kinetic factors.

3. A numerical thermodynamic simulation of a flow reaction system showed that almost complete transition of phosphorus to light gaseous substances $\left(P O, P_{2}\right)$ is thermodynamically possible at the temperatures that are close to the technological operation temperatures.

\section{Funding}

This work was supported by the program the Arctic - Scientific Fundamentals of New Technologies of Exploration, Conservation, and Development of the Russian Academy of Sciences, project no. 55.

\section{References}

[1] Tigunov L. P., Smirnov L. A., and Menadzhieva R. A. (2006). Manganese: Technology, Production, Application. Yekaterinburg: Izd. AMB. 
[2] Dashevskii V. Ya., Yusfin Yu. S., Polulyakh L. A., et al. (2016). Method of dephosphorization of manganese ores and concentrates. RF Patent 2594997. Byulleten Izobreteniy, no. 23.

[3] Kulikov I. S. (1975). Deoxidation of Metals. Moscow: Metallurgiya.

[4] Dashevskii V. Ya., Makeev D. B., Polulyakh L. A., Aleksandrov A. A., and Leont'ev L. I. (2017). Dephosphorization of Manganese-Containing Oxide Melts. Doklady Physical Chemistry, vol. 473, part 2, pp. 55-57. 